\title{
A P.C.F. Self-Similar Set with no Self-Similar Energy
}

\author{
Roberto Peirone \\ Università di Roma "Tor Vergata", Dipartimento di Matematica \\ via della Ricerca Scientifica, 00133, Roma, Italy \\ e.mail: peirone@mat.uniroma2.it
}

\begin{abstract}
A$ general class of finitely ramified fractals is that of P.C.F. self-similar sets. An important open problem in analysis on fractals was whether there exists a self-similar energy on every P.C.F. self-similar set. In this paper, I solve the problem, showing an example of a P.C.F. self-similar set where there exists no self-similar energy.
\end{abstract}

\section{Introduction.}

An important problem in analysis of fractals is the construction of a Laplace operator, or equivalently, an energy, more precisely, a self-similar Dirichlet form. The construction of a self-similar Dirichlet form has been investigated specially on finitely ramified fractals. Roughly speaking, a fractal is finitely ramified if the intersection of each pair of copies of the fractal is a finite set. The Sierpinski Gasket, the Vicsek Set and the Lindstrøm Snowflake are finitely ramified fractals, while the Sierpinski Carpet is not.

More specially, we consider the P.C.F. self similar-sets, a general class of finitely ramified fractals introduced by Kigami in [3]. A general theory with many examples can be found in [4]. On such a class of fractals, the basic tool used to construct a self-similar Dirichlet form is a discrete Dirichlet form defined on a special finite subset $V^{(0)}$ of the fractal. Such discrete Dirichlet forms have to be eigenforms, i.e. the eigenvectors of a special nonlinear operator $\Lambda_{r}$ called renormalization operator, which depends on a set of positive weights $r_{i}$ placed on the cells of the fractal. In [5], [10] and [6] criteria for the existence of an eigenform with prescribed weights are discussed. In particular, in [5], T. Lindstrøm proved that there exists an eigenform on the nested fractals with all weights equal to 1, C. Sabot in [10] proved a rather general criterion, and V. Metz in [6] improved the results in [10].

In [1], [7], [8] and [9], instead, the problem is considered whether on a given fractal there exists a $G$-eigenform. By this we mean a form $E$ which is an eigenform of the operator $\Lambda_{r}$ for some set of weights $r$. In such papers the existence of a G-eigenform was proved on some classes of P.C.F. self-similar sets. In fact, the following open problem is well-known

Does a G-eigenform exist on every P.C.F.self-similar set?

In this paper, I solve such a problem showing an example of P.C.F.self-similar set with no G-eigenform. Here, I consider a very general class of P.C.F. self-similar sets, as usually in my previous papers on this topic (see Section 2 for the details), but also in other papers (for example it is considered in [11]. The example is constructed in Section 3. It is a variant of the $N$-Gaskets, in the sense that every cell $V_{i}$ only intesects $V_{i-1}$ and $V_{i+1}$, and has twenty vertices. The ptoof is based on the evaluation of the effective conductivities on pairs of close vertices and of far vertices. Note that in [9] the existence of a G-eigenform is proved on every fractal (of the class considered here) but only if we consider the fractal 
generated by a set of similarities which is not necessarily the given set of similarities (see [9] for the details).

\section{Definitions and Notation.}

I will now define the fractal setting, which is based on that in [9]. This kind of approach was firstly given in [2]. We define a fractal by giving a fractal triple, i.e., a triple $\mathcal{F}:=$ $\left(V^{(0)}, V^{(1)}, \Psi\right)$ where $V^{(0)}=V$ and $V^{(1)}$ are finite sets with $\# V^{(0)} \geq 2$, and $\Psi$ is a finite set of one-to-one maps from $V^{(0)}$ into $V^{(1)}$ satisfying

$$
V^{(1)}=\bigcup_{\psi \in \Psi} \psi\left(V^{(0)}\right)
$$

We put $V^{(0)}=\left\{P_{1}, \ldots, P_{N}\right\}$, and of course $N \geq 2$. A set of the form $\psi\left(V^{(0)}\right)$ with $\psi \in \Psi$ will be called a cell or a 1-cell. We require that

a) For each $j=1, \ldots, N$ there exists a (unique) map $\psi_{j} \in \Psi$ such that $\psi_{j}\left(P_{j}\right)=P_{j}$, and $\Psi=\left\{\psi_{1}, \ldots, \psi_{k}\right\}$, with $k \geq N$.

b) $P_{j} \notin \psi_{i}\left(V^{(0)}\right)$ when $i \neq j$ (in other words, if $\psi_{i}\left(P_{h}\right)=P_{j}$ with $i=1, \ldots, k, j, h=$ $1, \ldots, N$, then $i=j=h$ ).

c) Any two points in $V^{(1)}$ can be connected by a path any edge of which belongs to a 1-cell, depending of the edge.

Of course, it immediately follows $V^{(0)} \subseteq V^{(1)}$. Let $\left.W=\right] 0,+\infty\left[^{k}\right.$ and put $V_{i}=\psi_{i}\left(V^{(0)}\right)$ for each $i=1, \ldots, k$. Let $J(=J(V))=\left\{\left\{j_{1}, j_{2}\right\}: j_{1}, j_{2}=1, \ldots, N, j_{1} \neq j_{2}\right\}$. It is well-known that on every fractal triple we can construct a P.C.F.-self-similar set.

We denote by $\mathcal{D}(\mathcal{F})$ or simply $\mathcal{D}$ the set of the Dirichlet forms on $V$, invariant with respect to an additive constant, i.e., the set of the functionals $E$ from $\mathbb{R}^{V}$ into $\mathbb{R}$ of the form

$$
E(u)=\sum_{\left\{j_{1}, j_{2}\right\} \in J} c_{\left\{j_{1}, j_{2}\right\}}(E)\left(u\left(P_{j_{1}}\right)-u\left(P_{j_{2}}\right)\right)^{2}
$$

with $c_{\left\{j_{1}, j_{2}\right\}}(E) \geq 0$. I will denote by $\widetilde{\mathcal{D}}(\mathcal{F})$ or simply $\widetilde{\mathcal{D}}$ the set of the irreducible Dirichlet forms, i.e., $E \in \widetilde{\mathcal{D}}$ if $E \in \mathcal{D}$ and moreover $E(u)=0$ if and only if $u$ is constant. The numbers $c_{\left\{j_{1}, j_{2}\right\}}(E)$ are called coefficients of $E$. We also say that $c_{\left\{j_{1}, j_{2}\right\}}(E)$ is the conductivity between $P_{j_{1}}$ and $P_{j_{2}}$ (with respect to $E$ ). Next, I recall the notion of effective conductivity. Let $E \in \widetilde{\mathcal{D}}$, and let $j_{1}, j_{2}=1, \ldots, N, j_{1} \neq j_{2}$. Then we put

$$
\mathcal{L}_{V ; j_{1}, j_{2}}=\left\{u \in \mathbb{R}^{V}: u\left(P_{j_{1}}\right)=0, u\left(P_{j_{2}}\right)=1\right\}
$$

It can be easily proved that the minimum $\min \left\{E(u): u \in \mathcal{L}_{V ; j_{1}, j_{2}}\right\}$ exists, is attained at a unique function, and amounts to $\min \left\{E(u): u \in \mathcal{L}_{V ; j_{2}, j_{1}}\right\}$. So, for $E \in \widetilde{\mathcal{D}}$ and $\left\{j_{1}, j_{2}\right\} \in J$, we define $\widetilde{C}_{\left\{j_{1}, j_{2}\right\}}(E)\left(=\widetilde{C}_{j_{1}, j_{2}}(E)\right)$ by

$$
\widetilde{C}_{\left\{j_{1}, j_{2}\right\}}(E)=\min \left\{E(u): u \in \mathcal{L}_{V ; j_{1}, j_{2}}\right\}=\min \left\{E(u): u \in \mathcal{L}_{V ; j_{2}, j_{1}}\right\}
$$


The value $\widetilde{C}_{\left\{j_{1}, j_{2}\right\}}(E)$ or short $\widetilde{C}_{\left\{j_{1}, j_{2}\right\}}$, is called effective conductivity between $P_{j_{1}}$ and $P_{j_{2}}$ (with respect to $E$ ). Note that $\widetilde{C}_{\left\{j_{1}, j_{2}\right\}}>0$. The following remark can be easily verified (see Remark 2.9 in [9].

Remark 2.1. If $j_{1}, j_{2}=1, \ldots, N, j_{1} \neq j_{2}$, and $E \in \widetilde{\mathcal{D}}$, then

$$
\min \left\{E(u): u \in \mathbb{R}^{V^{(0)}}: u\left(P_{j_{1}}\right)=t_{1}, u\left(P_{j_{2}}\right)=t_{2}\right\}=\left(t_{1}-t_{2}\right)^{2} \widetilde{C}_{\left\{j_{1}, j_{2}\right\}} .
$$

Recall that for every $r \in W:=] 0,+\infty\left[{ }^{k},\left(r_{i}:=r(i)\right)\right.$ the renormalization operator is defined as follows: for every $E \in \widetilde{\mathcal{D}}$ and every $u \in \mathbb{R}^{V^{(0)}}$,

$$
\begin{gathered}
\Lambda_{r}(E)(u)=\inf \left\{S_{1, r}(E)(v), v \in \mathcal{L}(u)\right\}, \\
S_{1, r}(E)(v):=\sum_{i=1}^{k} r_{i} E\left(v \circ \psi_{i}\right), \quad \mathcal{L}(u):=\left\{v \in \mathbb{R}^{V^{(1)}}: v=u \text { on } V^{(0)}\right\} .
\end{gathered}
$$

It is well known that $\Lambda_{r}(E) \in \widetilde{\mathcal{D}}$ and that the infimum is attained at a unique function $v:=H_{1, E ; r}(u)$. When $r \in W$, an element $E$ of $\widetilde{\mathcal{D}}$ is said to be an $r$-eigenform with eigenvalue $\rho>0$ if $\Lambda_{r}(E)=\rho E$. As this amounts to $\Lambda_{\frac{r}{\rho}}(E)=E$, we could also require $\rho=1$. The problem discussed in the present paper is that of the existence of a G-eigenform in $\widetilde{\mathcal{D}}$, in other words, the existence of $E \in \widetilde{\mathcal{D}}$ such that $\Lambda_{r}(E)=\rho E$ for some $\rho>0$ and $r \in W$. In next section, I will describe an example of a fractal triple where there exists no G-eigenform. To this aim, it will be useful the following standard lemma (see e.g., Lemma 3.3 in $[9]$.

Lemma 2.2 For every $E \in \widetilde{\mathcal{D}}$ and $\left\{j_{1}, j_{2}\right\} \in J$ we have

$$
\begin{gathered}
\widetilde{C}_{\left\{j_{1}, j_{2}\right\}}\left(\Lambda_{r}(E)\right)=\min \left\{S_{1, r}(E)(v): v \in H_{j_{1}, j_{2}}\right\}, \\
\text { where } H_{j_{1}, j_{2}}=\left\{v \in \mathbb{R}^{V^{(1)}}: v\left(P_{j_{1}}\right)=0, v\left(P_{j_{2}}\right)=1\right\} .
\end{gathered}
$$

\section{The Example.}

Let $\overline{\mathcal{F}}=\left(V^{(0)}, V^{(1)}, \Psi\right)$ be a fractal triple so defined. Let $N=2 N^{\prime}$ be a positive even number. Let $V^{(0)}=\left\{P_{1}, \ldots, P_{N}\right\}$, and we fix $N^{\prime}=10$ so $N=20$. Let $\Psi=\left\{\psi_{1}, \ldots, \psi_{20}\right\}$. Here, thus, $N=k=20$. In the following, the indices of the points and of the maps will be meant to be $\bmod 20$. For example, $i+9=2$ if $i=13$. Suppose $V_{i} \cap V_{i^{\prime}}=\varnothing$ if $i^{\prime} \notin\{i, i-1, i+1\}$, and

$$
V_{i} \cap V_{i+1}=\left\{\bar{Q}_{i}\right\}, \quad \bar{Q}_{i}=\left\{\begin{array}{l}
\psi_{i}\left(P_{i+1}\right)=\psi_{i+1}\left(P_{i}\right) \quad \text { if } i \text { odd } \\
\psi_{i}\left(P_{i+9}\right)=\psi_{i+1}\left(P_{i-8}\right) \quad \text { if } i \text { even. }
\end{array}\right.
$$

In this way $\bar{Q}_{i-1}=\psi_{i}\left(P_{\sigma(i)}\right), \bar{Q}_{i}=\psi_{i}\left(P_{\sigma(i)+10}\right)$, with $\sigma(i)=\left\{\begin{array}{l}i-1 \text { if } i \text { even, } \\ i-9 \text { if } i \text { odd. }\end{array}\right.$ Thus the points $\bar{Q}_{i}$ and $\bar{Q}_{i-1}$ are opposite in $V_{i}$. Here we say that $P_{h}$ and $P_{h+10}$ are opposite in 
$V^{(0)}$ and that $\psi_{i}\left(P_{h}\right)$ and $\psi_{i}\left(P_{h+10}\right)$ are opposite in $V_{i}$. In order to prove Theorem 3.2, we could use arguments based on effective resistances in series, but, in order to avoid some slightly technical points, I prefer to give a direct proof. We need the following well known lemma.

Lemma 3.1. For every positive integer $n$ and every $b_{i}>0, i=1, \ldots, n$, we have

$$
\left(\sum_{i=1}^{n} b_{i}^{-1}\right)^{-1}=\min \left\{\sum_{i=1}^{n} b_{i} x_{i}^{2}: x \in Y_{n}\right\}, \quad Y_{n}:=\left\{x \in \mathbb{R}^{n}: \sum_{i=1}^{n} x_{i}=1\right\} \text {. }
$$

Proof. Let $f: \mathbb{R}^{n} \rightarrow \mathbb{R}$ be defined by $f(x)=\sum_{i=1}^{n} b_{i} x_{i}^{2}$. Since $f$ is continuous and $f(x) \longrightarrow_{x \rightarrow \infty}+\infty, f$ attains a minimum $m$ on the closed set $Y_{n}$ at some point $\bar{x}$. We find $\bar{x}$ using the Lagrange multiplier rule. We have $b_{i} \bar{x}_{i}=\lambda$ for some $\lambda \in \mathbb{R}$ and every $i=1, \ldots, n$. Thus, $1=\sum_{i=1}^{n} \bar{x}_{i}=\lambda \sum_{i=1}^{n} b_{i}^{-1}$, and $\bar{x}_{i}=\lambda b_{i}^{-1}=b_{i}^{-1}\left(\sum_{j=1}^{n} b_{j}^{-1}\right)^{-1}$. Since $m=f(\bar{x})$, a simple calculation completes the proof.

Theorem 3.2. On $\overline{\mathcal{F}}$ there exists no $G$-eigenform.

Proof. Suppose by contradiction there exist $E \in \widetilde{\mathcal{D}}$ and $r \in W$ such that $\Lambda_{r}(E)=E$. Of course, in view of Lemma 2.2, this implies

$$
\widetilde{C}_{\left\{j_{1}, j_{2}\right\}}(E)=\min \left\{S_{1, r}(E)(v): v \in H_{j_{1}, j_{2}}\right\} \quad \forall\left\{j_{1}, j_{2}\right\} \in J .
$$

Now, let $\bar{r}=\max \left\{\min \left\{r_{2 h+1}, r_{2 h+2}\right\}: h=0, \ldots, 9\right\}$. Thus,

$$
\begin{gathered}
\exists \bar{h}=0, \ldots, 9: \forall d=1,2: r_{2 \bar{h}+d} \geq \bar{r}, \\
\forall h=0, \ldots, 9 \exists d=1,2: r_{2 h+d} \leq \bar{r} .
\end{gathered}
$$

Next, we evaluate $\widetilde{C}_{2 \bar{h}+1,2 \bar{h}+2}(E)$ using (3.1). Let $v \in H_{2 \bar{h}+1,2 \bar{h}+2}$. Then, since by definition $S_{1, r}(E)(v)=\sum_{i=1}^{20} r_{i} E\left(v \circ \psi_{i}\right) \geq \sum_{d=1}^{2} r_{2 \bar{h}+d} E\left(v \circ \psi_{2 \bar{h}+d}\right)$, in view of (3.2) we have

$$
S_{1, r}(E)(v) \geq \bar{r}\left(E\left(v \circ \psi_{2 \bar{h}+1}\right)+E\left(v \circ \psi_{2 \bar{h}+2}\right)\right) .
$$

Let $t=v\left(\bar{Q}_{2 \bar{h}+1}\right)$. Let $v_{1}=v \circ \psi_{2 \bar{h}+1}, v_{2}=v \circ \psi_{2 \bar{h}+2}$. Then we have

$$
\begin{aligned}
& v_{1}\left(P_{2 \bar{h}+1}\right)=v\left(P_{2 \bar{h}+1}\right)=0, \quad v_{1}\left(P_{2 \bar{h}+2}\right)=v\left(\psi_{2 \bar{h}+1}\left(P_{2 \bar{h}+2}\right)\right)=v\left(\bar{Q}_{2 \bar{h}+1}\right)=t, \\
& v_{2}\left(P_{2 \bar{h}+2}\right)=v\left(P_{2 \bar{h}+2}\right)=1, \quad v_{2}\left(P_{2 \bar{h}+1}\right)=v\left(\psi_{2 \bar{h}+2}\left(P_{2 \bar{h}+1}\right)\right)=v\left(\bar{Q}_{2 \bar{h}+1}\right)=t .
\end{aligned}
$$

By Remark 2.1 and Lemma 3.1 with $n=2$, we thus have

$$
E\left(v \circ \psi_{2 \bar{h}+1}\right)+E\left(v \circ \psi_{2 \bar{h}+2}\right) \geq\left(t^{2}+(1-t)^{2}\right) \widetilde{C}_{2 \bar{h}+1,2 \bar{h}+2}(E) \geq \frac{1}{2} \widetilde{C}_{2 \bar{h}+1,2 \bar{h}+2}(E) .
$$


By (3.4) and (3.1) we have $\widetilde{C}_{2 \bar{h}+1,2 \bar{h}+2}(E) \geq \frac{\bar{r}}{2} \widetilde{C}_{2 \bar{h}+1,2 \bar{h}+2}(E)$, thus

$$
\bar{r} \leq 2
$$

Let now $\widehat{l}=1, \ldots, 20$ be so that

$$
\widetilde{C}_{\widehat{l, l+10}}(E) \geq \widetilde{C}_{l, l+10}(E) \quad \forall l=1, \ldots, 20
$$

Note that, in view of Remark 2.1, for every $i=1, \ldots, 20$ and every $t_{1}, t_{2} \in \mathbb{R}$ there exists $u_{i, t_{1}, t_{2}} \in \mathbb{R}^{V^{(0)}}$ such that

$$
u_{i, t_{1}, t_{2}}\left(P_{i}\right)=t_{1}, u_{i, t_{1}, t_{2}}\left(P_{i+10}\right)=t_{2}, E\left(u_{i, t_{1}, t_{2}}\right)=\left(t_{1}-t_{2}\right)^{2} \widetilde{C}_{i, i+10}(E) .
$$

Next, define $v \in H_{\widehat{l, ~},+10}$, in terms of $x, x^{\prime} \in Y_{9}$. Given such $x, x^{\prime}$ let $s(n)=\sum_{i=1}^{n} x_{i}$, $s^{\prime}(n)=1-\sum_{i=1}^{n} x_{i}^{\prime}$ for $n=0, \ldots, 9$. Let $v \in \mathbb{R}^{V^{(1)}}$ be so that

$$
v \circ \psi_{i}= \begin{cases}0 & \text { if } i=\widehat{l} \\ 1 & \text { if } i=\widehat{l}+10 \\ u_{\sigma(i), s(i-\widehat{l}-1), s(i-\widehat{l})} & \text { if } i=\widehat{l}+1, \ldots, \widehat{l}+9, \\ u_{\sigma(i), s^{\prime}(i-\widehat{l}-11), s^{\prime}(i-\widehat{l}-10)} & \text { if } i=\widehat{l}+11, \ldots, \widehat{l}+19 .\end{cases}
$$

We easily see that the definition of $v$ is correct, i.e., the definition of $v$ at the points $\bar{Q}_{i}$ (the only points lying in different cells) is independent of the two representations of $\bar{Q}_{i}$, that is, $\bar{Q}_{i}=\psi_{i}\left(P_{\sigma(i)+10}\right)$ and $\bar{Q}_{i}=\psi_{i+1}\left(P_{\sigma(i+1)}\right)$. Moreover, as $P_{\widehat{l}}=\psi_{\widehat{l}}\left(P_{\widehat{l}}\right)$, and $P_{\widehat{l}+10}=$ $\psi_{\widehat{l}+10}\left(P_{\widehat{l}+10}\right)$. we immediately see that $v \in H_{\widehat{l}, \vec{l}+10}$. Since $E\left(v \circ \psi_{\widehat{l}}\right)=E\left(v \circ \psi_{\widehat{l}+10}\right)=0$, we have

$$
\begin{aligned}
& S_{1, r}(E)(v)=\sum_{i=\widehat{l}}^{\widehat{l}+19} r_{i} E\left(v \circ \psi_{i}\right)=\left(\sum_{i=\widehat{l}+1}^{\widehat{l}+9} r_{i} E\left(v \circ \psi_{i}\right)+\sum_{i=\widehat{l}+11}^{\widehat{l}+19} r_{i} E\left(v \circ \psi_{i}\right)\right) \\
& =\sum_{i=\widehat{l}+1}^{\widehat{l}+9} r_{i} E\left(u_{\sigma(i), s(i-\widehat{l}-1), s(i-\widehat{l})}\right)+\sum_{i=\widehat{l}+11}^{\widehat{l}+19} r_{i} E\left(u_{\sigma(i), s^{\prime}(i-\widehat{l}-11), s^{\prime}(i-\widehat{l}-10)}\right) \\
& =\sum_{i=\widehat{l}+1} r_{i} \widetilde{C}_{\sigma(i), \sigma(i+10)} x_{i-\widehat{l}}^{2}+\sum_{i=\widehat{l}+11} r_{i} \widetilde{C}_{\sigma(i), \sigma(i+10)}\left(x_{i-\widehat{l}-10}^{\prime}\right)^{2} \\
& =\sum_{i=1}^{9} r_{i+\widehat{l}} \widetilde{C}_{\sigma(i+\widehat{l}), \sigma(i+\widehat{l})+10} x_{i}^{2}+\sum_{i=1}^{9} r_{i+\widehat{l}+10} \widetilde{C}_{\sigma(i+\widehat{l}+10), \sigma(i+\widehat{l}+10)+10}\left(x_{i}^{\prime}\right)^{2}
\end{aligned}
$$




$$
\leq \widetilde{C}_{\widehat{l}, \widehat{l}+10}(E)\left(\sum_{i=1}^{9} r_{i+\widehat{l}} x_{i}^{2}+\sum_{i=1}^{9} r_{i+\widehat{l}+10}\left(x_{i}^{\prime}\right)^{2}\right) .
$$

So far we have taken arbitrary $x, x^{\prime} \in Y_{9}$. Now take those $x, x^{\prime} \in Y_{9}$ that minimize the sums in previous formula. By Lemma 3.1, with this $v$ we have

$$
S_{1, r}(E)(v) \leq \widetilde{C}_{\widehat{l}, \widehat{l}+10}(E)\left(\left(\sum_{i=1}^{9}\left(r_{i+\widehat{l}}\right)^{-1}\right)^{-1}+\left(\sum_{i=1}^{9}\left(r_{i+\widehat{l}+10}\right)^{-1}\right)^{-1}\right)
$$

By (3.3), for at least four $i=1, \ldots, 9$ we have $r_{i+\widehat{l}} \leq \bar{r}$, so $\left(\sum_{i=1}^{9}\left(r_{i+\widehat{l}}\right)^{-1}\right)^{-1}<\frac{\bar{r}}{4}$. Similarly, $\left(\sum_{i=1}^{9}\left(r_{i+\widehat{l}+10}\right)^{-1}\right)^{-1}<\frac{\bar{r}}{4}$. Since $v \in H_{\widehat{l}, \widehat{l}+10}$, by (3.8) and (3.1) we have

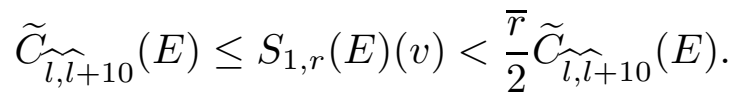

Thus, $\bar{r}>2$, which contradicts (3.5).

\section{References}

[1] B.M. Hambly, V. Metz, A. Teplyaev, Self-similar energies on post-critically finite selfsimilar fractals, J. London Math Soc. (2) 74, pp. 93-112, 2006

[2] K.Hattori, T. Hattori, H. Watanabe, Gaussian field theories on general networks and the spectral dimension, Progr. Theoret. Phys. Suppl. 92, pp. 108-143, 1987

[3] J. Kigami, Harmonic calculus on p.c.f. self-similar sets, Trans. Amer. Math. Soc. 335, pp.721-755, 1993

[4] J. Kigami, Analysis on fractals, Cambridge University Press, 2001

[5] T. Lindström, Brownian motion on nested fractals, Mem. Amer. Math. Soc. 83 No. 420, 1990

[6] V. Metz, The short-cut test, J. Funct. Anal. 220, pp. 118-156, 2005

[7] R. Peirone, Existence of eigenforms on fractals with three vertices, Proc. Royal Soc. Edinburgh Sect. A 137, 2007

[8] R. Peirone, Existence of eigenforms on nicely separated fractals, in Analysis of graphs and its applications, Amer. Math. Soc., Providence, pp. 231-241, 2008

[9] R. Peirone, Existence of self-similar energies on finitely ramified fractals, Journal d'Analyse Mathmatique Volume 123 Issue 1, pp. 35-94, 2014

[10] C. Sabot, Existence and uniqueness of diffusions on finitely ramified self-similar fractals, Ann. Sci. École Norm. Sup. (4) 30, pp. 605-673, 1997

[11] R.S. Strichartz, Differential equations on fractals: a tutorial, Princeton University Press, 2006 